\title{
Severe Asthma in a General Population Study: Prevalence and Clinical Characteristics
}

\author{
Lina Rönnebjerg (1D' \\ Malin Axelsson (D) ${ }^{2}$ \\ Hannu Kankaanranta (iD) 1,3,4 \\ Helena Backman (D) ${ }^{5}$ \\ Madeleine Rådinger (iD) \\ Bo Lundbäck (D)' \\ Linda Ekerljung (D) \\ 'Krefting Research Centre, Department \\ of Internal Medicine and Clinical \\ Nutrition, Institute of Medicine, \\ University of Gothenburg, Gothenburg, \\ Sweden; ${ }^{2}$ Department of Care Science, \\ Faculty of Health and Society, Malmö \\ University, Malmö, Sweden; ${ }^{3}$ Department \\ of Respiratory Medicine, Seinäjoki \\ Central Hospital, Seinäjoki, Finland; \\ ${ }^{4}$ Tampere University Respiratory \\ Research Group, Faculty of Medicine and \\ Health Technology, University of \\ Tampere, Tampere, Finland; ${ }^{5}$ Department \\ of Public Health and Clinical Medicine, \\ Umeå University, Umeå, Sweden
}

Correspondence: Lina Rönnebjerg Krefting Research Centre, Department of Internal Medicine and Clinical Nutrition, Institute of Medicine, University of Gothenburg, Box 424, 405 30,

Gothenburg, Sweden

Tel +4631786 6709

Email lina.ronnebjerg@gu.se
Purpose: Current guidelines primarily use medication levels to distinguish severe asthma from other types of asthma. In addition, severe asthma must also be uncontrolled at highintensity treatment or become uncontrolled if treatment level is decreased. To date, only a few studies have used this definition to investigate the prevalence and clinical characteristics of severe asthma in population-based samples. Therefore, the aim of this study was to evaluate the prevalence and clinical characteristics of individuals with severe asthma in the population-representative West Sweden Asthma Study.

Materials and Methods: In this cross-sectional population-based study, a randomly selected sample $(n=1172)$ and a separate asthma sample $(n=744)$ underwent clinical examinations, completed a structured interview and responded to questionnaires. Severe asthma was defined as at least one feature of uncontrolled asthma despite treatment in line with the Global Initiative for Asthma (GINA) steps 4/5. This treatment level required a minimum medium dose of inhaled corticosteroids (ICS) plus a second controller or oral corticosteroids. Results: The prevalence of severe asthma was $1.1 \%$ in the adult random sample and $9.5 \%$ within the asthma sample. Individuals with severe asthma were older and had more symptoms, activity limitations, heart disease and blood neutrophils compared to those with other asthma. They also had lower lung function and despite these impairments, 32\% did not have annual contact with a healthcare provider.

Conclusion: The prevalence of severe asthma was higher compared to previous studies, and many individuals with severe asthma did not have regular contact with healthcare providers. Due to the high burden of symptoms and impairments for individuals with severe asthma, it is important that the healthcare system implement strategies to improve follow-up and evaluate these patients according to existing guidelines.

Keywords: asthma epidemiology, asthma control, asthma-medication, asthma severity symptoms, lung function

\section{Introduction}

A limited but significant group of individuals with asthma does not achieve full asthma control despite higher doses of inhaled corticosteroids (ICS), which is the primary controller treatment in asthma. ${ }^{1,2}$ Those with severe asthma have more symptoms, exacerbations and comorbidities compared to those with mild-to-moderate asthma. ${ }^{3-5}$ They also have lower asthma control, quality of life and lung function despite high doses of ICS. ${ }^{3,6-8}$ As the severity of asthma increases, so do the costs for society, ${ }^{9,10}$ and severe uncontrolled asthma accounts for $60 \%$ of all asthma costs. ${ }^{11}$

The current definition by the European Respiratory Society/American Thoracic Society (ERS/ATS) primarily uses medication levels to distinguish severe asthma from other asthma. ${ }^{1}$ Severe asthma requires high daily doses of ICS plus a second controller 
or oral corticosteroids to maintain asthma control or is still uncontrolled despite this treatment. ${ }^{1}$ The Global Initiative for Asthma (GINA) suggests that severe asthma can be defined by both medium and high doses of ICS, if used in conjunction with a second controller or oral corticosteroids (corresponding to GINA treatment steps 4/5). ${ }^{2}$ Although there are some differences among previous studies regarding ICS dose and asthma control requirements, the prevalence of severe asthma has been estimated at $4-8 \%{ }^{5,6,12-16}$ within asthma samples from clinics, ${ }^{14}$ registries ${ }^{6,12,13,15,16}$ and from one population-based study. ${ }^{5}$ Data on severe asthma prevalence at the population level is more limited, but indicates a prevalence of about $0.5 \% .^{5,6,12}$ However, studies on the clinical characteristics of severe asthma are scarce in population-representative samples, ${ }^{5}$ and one obstacle is the preference for thorough clinical evaluation before determining severe asthma. ${ }^{1}$ This evaluation requires at least three months of treatment optimization (inhaler technique and adherence) and management of trigger factors and comorbidities, $1,2,17$ which is not plausible in most population-based studies. In addition, the need for multiple visits increases the risk of sampling bias and capturing the prevalence in the general population becomes more difficult. Consequently, to get a more representative understanding of severe asthma we aimed to evaluate the prevalence and clinical characteristics of individuals with severe asthma in a large population-based cohort.

\section{Materials and Methods}

\section{Study Population}

The cross-sectional West Sweden Asthma Study (WSAS) has been described thoroughly in other studies. ${ }^{18-20}$ In brief, WSAS was initiated with a postal survey in 2008, with clinical follow-up from 2009-2012. The survey was sent to 30,000 randomly selected inhabitants (age 16-75 years) in west Sweden, sampled from the comprehensive population register, and 18,087 responded. To improve comparability to the general population in the area, a nonresponder study was conducted after the survey, showing high representativeness. ${ }^{21}$

Among responders, a randomly selected sample $(n=2000)$ was invited to participate in extensive clinical examinations, a structured interview and self-administered questionnaires. In addition to the random sample, all responders with asthma $(n=1524)$ were also invited to participate in the same procedures. Of the 2000 invited participants in the random sample, 1172 (59\%) participated in the clinical examinations; of the 1524 invited from the asthma sample, 834 (55\%) participated. After the visit, 228 were excluded from the asthma sample because they did not have current asthma (please see Supplementary Information 02 for definitions). Thus, 744 participants with current asthma from both samples (138 +606 ) were included in the present study (Figure 1). The Regional Ethical Review Board in Gothenburg, Sweden, approved the study (DNR: 593-08, 034-08) and was conducted in accordance with the Declaration of Helsinki. ${ }^{22}$ All participants provided written informed consent.

\section{Data Collection}

The structured interview included questions about respiratory and allergic diseases and symptoms, comorbidities, use of pharmacological treatment, asthma control according to GINA 2009 (the most current version of the guidelines at the time), ${ }^{23}$ healthcare use and possible determinants of respiratory disease. Adherence to treatment with ICS was assessed during this interview: "daily intake" corresponds to the best possible option 'most days' as the response to how often the ICS and the second controller (or ICS/long-acting beta ${ }_{2}$-agonist [LABA]) was taken. The self-administered questionnaires included questions about the participant's asthma management and the Asthma Control Test (ACT). ${ }^{24}$

The clinical examinations included spirometry, reversibility testing, methacholine challenge test, vital signs, anthropometric measurements and blood samples for differential cell count and specific circulating IgE levels (see Supplementary Information 01 for details). Skin prick testing for eleven common inhalant allergens was performed after withholding antihistamines for $\geq 72$ hours.

\section{Definitions}

Severe asthma: Current asthma (please see Supplementary Information 02 for a complete list of definitions) and use of asthma medication treatment according to the GINA treatment steps four or five; ie, daily intake of medium-tohigh doses of ICS plus a second controller or oral corticosteroids. Medium dose refers to $>400 \mu \mathrm{g}$ of budesonide or equivalent. ${ }^{2}$ In addition, uncontrolled asthma as per the ERS/ATS guidelines from $2014^{1}$ was required for the group of severe asthma.

Other asthma: Current asthma that did not meet requirements for severe asthma.

Uncontrolled asthma by ERS/ATS 2014: Any feature of the following was categorized as uncontrolled 


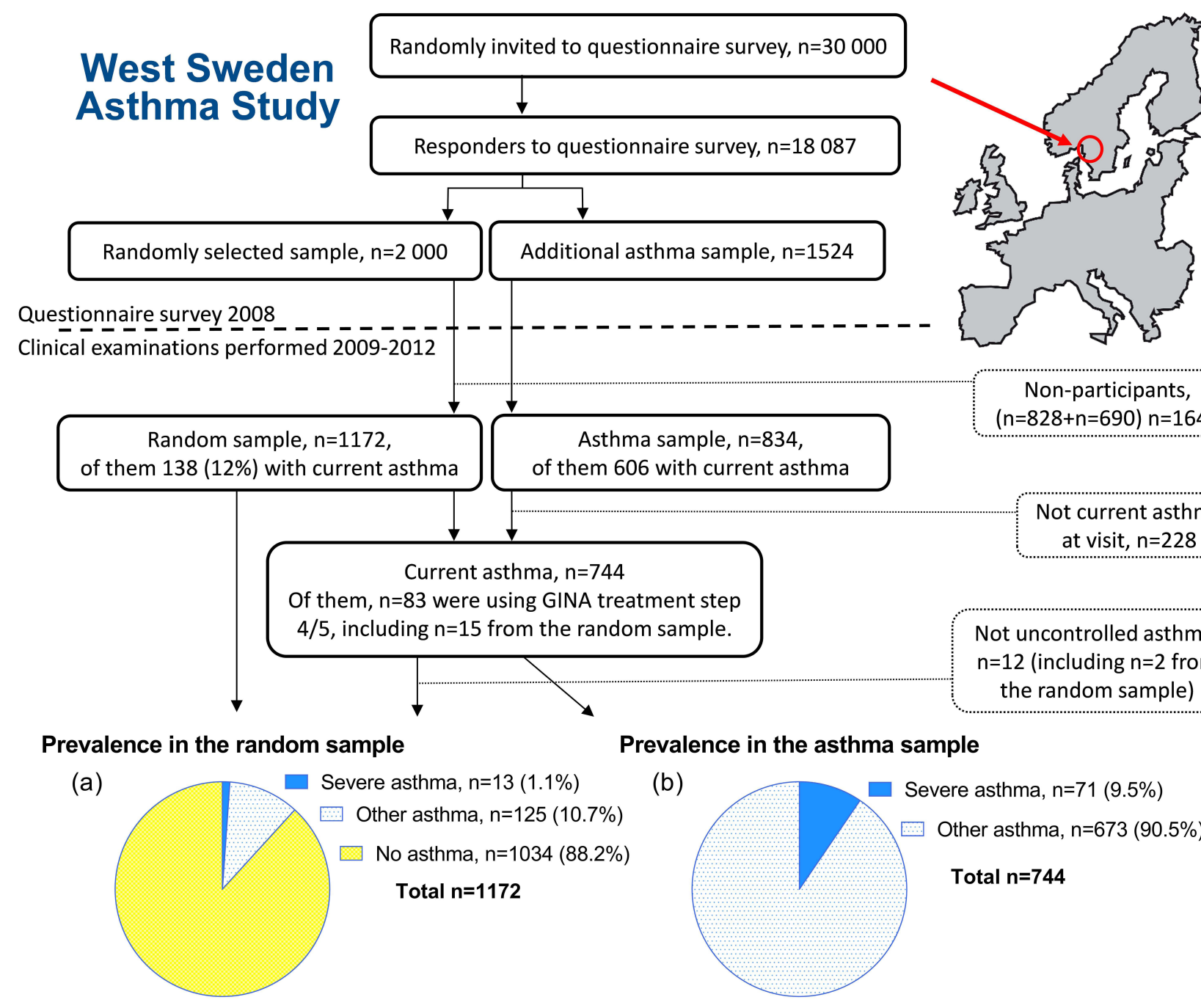

Figure I Flowchart with sampling procedure of the study population and prevalence of severe, other and no asthma in the random sample (A) and prevalence of severe and other asthma in the asthma sample (B). Image of Europe and West Sweden made by Anders Euren, University of Gothenburg.

asthma: 1) poor symptom control: ACT-score of $<20$ or "not well controlled" by GINA guidelines (daytime symptoms more than twice a week or activity limitations last four weeks or nocturnal symptoms/awakening last four weeks or need for reliever/rescue treatment more than twice a week), 2) frequent exacerbations: two or more exacerbations with use of oral corticosteroids in the previous year, 3) serious exacerbations: at least one emergency visit or hospitalization in the previous year due to asthma or 4) low lung function (Forced Expiratory Volume in one second $\left[\mathrm{FEV}_{1}\right]<80 \%$ of predicted). ${ }^{1}$

\section{Statistical Analyses}

Calculations of prevalence were made both in the random sample $(n=1172)$ and in the current asthma sample $(n=744)$. Clinical characteristics of severe and other asthma were analyzed only in the current asthma sample.

Statistical analyses were performed using SPSS version 25 (IBM Corp, New York, USA). Most continuous variables (except Forced Expiratory Capacity [FVC]) were non-normally distributed and groups of severe and other asthma were compared using the Mann-Whitney $U$-test. FVC values were also compared using the two-tailed independent samples $t$-test. Proportions were compared using Chi-squared tests. $P$-values were calculated with Fisher's exact test to compare proportions of nominal data with two categories, Pearson's chi-squared test when comparing proportions of nominal data with more than two categories, and Mantel-Haenszel test for trend when comparing proportions of ordinal data between groups. $P<0.05$ was considered statistically significant. 


\section{Results}

Prevalence Based on Medication Use and Asthma Control

Based on medication use only, 15 individuals (1.3\%) in the random sample $(\mathrm{n}=1172$, median age 52.1 years, $53.8 \%$ women) and 83 individuals (11.2\%) in the current asthma sample ( $n=744$, median age 48.0 years, $61.8 \%$ women) used asthma medication treatment in line with GINA step 4/5. Within the current asthma sample, 12 individuals did not meet requirements for uncontrolled asthma by ERS/ATS 2014 (of which two also were part of the random sample) and were thereby categorized as other asthma. Accordingly, the prevalence of severe asthma in the current study was estimated at $1.1 \%(n=13)$ in the random sample and $9.5 \%$ $(\mathrm{n}=71$, Figure 1) within the current asthma sample. Among the 71 individuals with severe asthma, 29.6\% $(n=21$, corresponding to $2.8 \%$ of current asthma) used GINA step 5 treatment and $70.4 \%(n=50,6.7 \%$ of current asthma) used step 4 treatment.

Individuals with severe asthma used more rescue medication (short-acting beta -agonist $_{\text {[SABA] }}$ compared to individuals with other asthma, 42.3\% used SABA most days, compared to $12.5 \%$ among those with other asthma $(p<0.001)$. Oral corticosteroids and/or antibiotics were used in the last year by $35.8 \%$ in the severe asthma group and $12.5 \%$ in the other asthma group $(p<0.001)$.

\section{Demographics of Severe Asthma versus Other Asthma}

Individuals with severe asthma were older (median age: 59.2 years compared to 46.9 years $[p<0.001])$ and they were diagnosed with asthma later compared to those with other asthma (Table 1). The proportion of individuals with asthma onset at 40 years or later was $29.6 \%$ among those with severe asthma compared to $13.7 \%$ among other asthma $(p=0.002)$. Self-reported heart disease such as heart failure and angina pectoris and use of $\geq 1$ medication for heart disease were more common among those with severe asthma compared to other asthma (Table 1). Individuals with severe asthma were less atopic and tended to have a higher number of packyears (median 18.0 vs $10.0, p=0.084$ ). There were no significant differences in gender, nasal polyps, BMI or exposure to gas, dust and fumes (Table 1). Among the 71 individuals with severe asthma, $28.2 \%(\mathrm{n}=20)$ had a smoking history of $>10$ pack-years and $\mathrm{FEV}_{1} / \mathrm{FVC}<0.7$ compared to $7.4 \%$ among other asthma $(p<0.001)$.

\section{Clinical Characteristics}

Many symptoms were more common among individuals with severe asthma compared to individuals with other asthma (Table 2). More than half of those with severe asthma experienced wheezing (recurrently) when breathing, $52.1 \%$ compared to $36.3 \%$ among those with other asthma $(p=0.010)$. Dyspnea was also more common where $36.2 \%$ of those with severe asthma got breathless from walking at their own pace on the level, while the corresponding proportion for those with other asthma was $10.9 \%(p<0.001$, Table 2$)$.

Lung function was poorer in individuals with severe asthma compared to other asthma (Figure 2). Median $\mathrm{FEV}_{1}$ was $81 \%$ (IQR $67-100 \%$ ) of predicted for individuals with severe asthma and 99\% (IQR $89-108 \%$ ) of predicted for those with other asthma $(p<0.001)$. To enable comparison to other studies, the corresponding mean values for $\mathrm{FEV}_{1}$ were $81 \%$ and $98 \%$ of predicted, respectively. The median $\mathrm{FEV}_{1} / \mathrm{FVC}$ was 0.72 (IQR 0.61-0.79) for severe asthma and 0.79 (IQR 0.73-0.83) for other asthma $(p<0.001)$. More individuals with severe asthma had a positive reversibility test than those with other asthma, even though none withheld their ICS prior to examination (Figure 2). The methacholine challenge test did not show any significant differences between the two groups (see Supplementary Information 03 for details). Unfortunately, $60.6 \%(\mathrm{n}=43)$ of persons with severe asthma could not perform the test due to low lung function ( $\mathrm{FEV}_{1}<70 \%$ ), high age ( $>60$ years) or use of heart medications. The corresponding proportion for other asthma was lower than for severe asthma, at $46.8 \%(\mathrm{n}=308$, $p=0.033$ ).

Individuals with severe asthma had significantly higher counts of blood neutrophils, but only a non-significant trend of higher counts of eosinophils. There was no significant difference between the groups when different cutoff values were used for eosinophil count, but higher neutrophil levels (using thresholds of $\geq 3.0\left(\times 10^{9} / \mathrm{L}\right)$ and higher) were consistently more common in severe asthma than in other asthma (Table 3).

\section{Impact on Daily Life}

Among individuals with severe asthma, 38.8\% had daily symptoms compared to $15.3 \%$ of those with other asthma $(p<0.001)$, and more with severe asthma experienced night waking due to asthma compared to those with other asthma. Four times as many individuals with severe 
Table I Baseline Characteristics Among Individuals with Severe vs Other Asthma

\begin{tabular}{|c|c|c|c|c|}
\hline Characteristics & & Severe Asthma & Other Asthma & p-value \\
\hline Total $n=744$ & & $n=71$ & $n=673$ & \\
\hline Women & $\%$ & 69.0 & 61.1 & 0.202 \\
\hline Age (Min-Max: 19-78 years) & median (IQR) & $59.2(44.7-68.6)$ & $46.9(35.4-59.5)$ & $<0.001$ \\
\hline Age of asthma onset & median (IQR) & $20.0(9.0-42.0)$ & I5.5 (7.0-30.0) & 0.026 \\
\hline Asthma onset, later than 40 years & $\%$ & 29.6 & 13.7 & 0.002 \\
\hline BMI $\left(\mathrm{kg} / \mathrm{m}^{2}\right)$ & median (IQR) & $27.7(24.1-32.2)$ & $26.4(23.8-29.6)$ & 0.112 \\
\hline Waist circumference $(\mathrm{cm})$ & median (IQR) & $96.5(86.0-108.0)$ & $91.0(83.0-101.8)$ & 0.023 \\
\hline Atopic & $\%$ & 40.3 & 57.7 & 0.007 \\
\hline Allergic or chronic rhinitis & $\%$ & 81.7 & 83.1 & 0.742 \\
\hline \multicolumn{5}{|l|}{ Comorbidity } \\
\hline Nasal polyps & $\%$ & 19.7 & 16.3 & 0.502 \\
\hline Angina pectoris & $\%$ & 8.5 & 1.8 & 0.005 \\
\hline Myocardial infarction & $\%$ & 4.2 & 1.0 & 0.061 \\
\hline Heart failure & $\%$ & 7.0 & 0.4 & $<0.001$ \\
\hline Arrhythmias & $\%$ & 7.0 & 5.3 & 0.580 \\
\hline Other heart disease & $\%$ & 1.4 & 2.4 & 1.000 \\
\hline$\geq 1$ medication related to heart disease & $\%$ & 26.8 & 8.6 & $<0.001$ \\
\hline Smoking status & & & & 0.341 \\
\hline Never smoker & $\%$ & 46.5 & 55.1 & \\
\hline Former smoker & $\%$ & 33.8 & 26.6 & \\
\hline Current smoker & $\%$ & 19.7 & 18.3 & \\
\hline \multicolumn{5}{|l|}{ Smoking history } \\
\hline Pack-years & median (IQR) & $18.0(4.0-36.0)$ & $10.0(4.0-22.0)$ & 0.084 \\
\hline Pack-years $>10$ years & $\%$ & 38.0 & 25.6 & 0.075 \\
\hline Exposed to gas, dust or fumes at work & $\%$ & 33.8 & 28.7 & 0.141 \\
\hline $\begin{array}{l}\text { Family history of asthma, allergy or } \\
\text { both }\end{array}$ & & & & 0.502 \\
\hline History of both & $\%$ & 23.9 & 26.6 & \\
\hline History of asthma & $\%$ & 4.2 & 7.4 & \\
\hline History of allergy & $\%$ & 18.3 & 19.8 & \\
\hline No history & $\%$ & 38.0 & 36.6 & \\
\hline Level of education & & & & $0.045^{a}$ \\
\hline Compulsory school $\leq 9$ years & $\%$ & 33.3 & 19.6 & \\
\hline Upper secondary school $\leq 4$ years & $\%$ & 30.4 & 38.7 & \\
\hline University $\geq 1$ year & $\%$ & 36.2 & 41.8 & \\
\hline
\end{tabular}

Notes: ${ }^{a}$ Mantel-Haenszel test for trend. Bold figures indicate statistical significance.

asthma (29.6\%) had two or more exacerbations the last 12 months compared to those with other asthma (7.3\%; $p<0.001)$. Emergency visits and hospitalizations were more common in severe than in other asthma, but $31.7 \%$ of those with severe asthma did not have annual contact with a healthcare provider due to their asthma (Figure 3).
By definition, all with severe asthma had uncontrolled asthma according to the ERS/ATS 2014. ${ }^{1}$ Correspondingly, asthma control was poorer among those with severe asthma than in other asthma, not only when asthma control was defined by ERS/ATS but also by GINA and ACT (Figure 3). Median ACT score was 20.0 
Table 2 Prevalence of Respiratory Symptoms Among Individuals with Severe vs Other Asthma

\begin{tabular}{|l|c|c|c|c|}
\hline Symptoms $^{\mathbf{a}}$ & & $\begin{array}{c}\text { Severe } \\
\text { Asthma }\end{array}$ & $\begin{array}{c}\text { Other } \\
\text { Asthma }\end{array}$ & p-value \\
\hline Total $\mathrm{n}=744$ & & $\mathrm{n}=71$ & $\mathrm{n}=673$ & \\
Any wheeze, ever & $\%$ & 97.2 & 94.5 & 0.572 \\
Any wheeze & $\%$ & 83.1 & 79.0 & 0.537 \\
Asthmatic wheeze & $\%$ & 64.8 & 47.7 & $\mathbf{0 . 0 0 8}$ \\
Recurrent wheeze & $\%$ & 52.1 & 36.3 & $\mathbf{0 . 0 1 0}$ \\
Persistent wheeze & $\%$ & 23.7 & 12.8 & $\mathbf{0 . 0 1 5}$ \\
Attacks of shortness of & $\%$ & 50.7 & 38.9 & 0.057 \\
breath & & & & \\
Morning cough & $\%$ & 53.3 & 41.3 & $\mathbf{0 . 0 5 8}$ \\
Longstanding cough & $\%$ & 60.6 & 29.6 & $<\mathbf{0 . 0 0 1}$ \\
Sputum production & $\%$ & 62.0 & 33.3 & $<\mathbf{0 . 0 0 1}$ \\
Dyspnea mMRC $\geq 2^{\mathrm{b}}$ & $\%$ & 36.2 & 10.9 & $<\mathbf{0 . 0 0 1}$ \\
\hline
\end{tabular}

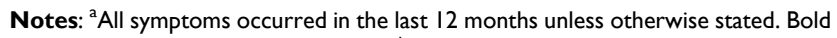
figures indicate statistical significance. ${ }^{b} \mathrm{Grade}$ two or higher corresponds to the option where an individual gets breathless from walking at their own pace on the level or from less activity.

Abbreviation: mMRC, modified Medical Research Council dyspnoea scale.

(IQR 15.0-22.0) among individuals with severe asthma and 22.0 (IQR 19.0-24.0) among those with other asthma $(p<0.001)$.

\section{Discussion}

In our population-representative sample of adults, the prevalence of severe asthma was $1.1 \%$. Within the current asthma sample, the prevalence of severe asthma was $9.5 \%$. Participants with severe asthma had more impairments in daily life, such as activity limitations, exacerbations and more symptoms. They were also older, less atopic, had lower lung function and higher levels of blood neutrophils compared to those with other asthma. Despite these impairments, one-third did not have annual contact with a healthcare provider.

Ever since the medication levels became the basis of the definition of severe asthma, studies reporting prevalence have used different cut-offs for ICS dose. ${ }^{5,6,12-16,25,26}$ Some studies have reported the prevalence of severe asthma defined as uncontrolled asthma despite high dose ICS (ie, refractory asthma). ${ }^{12,25,26}$ Others have presented controlled and uncontrolled asthma together under the umbrella of severe asthma, ${ }^{5,6,13-15}$ or as a mix of the methods. ${ }^{16}$ With these differences in mind, the prevalence of severe asthma among asthmatics, was estimated to be $4-8 \%, 6,12,13,15,16$ ranging from $0.9 \%{ }^{26}$ to $8.1 \%{ }^{6}$ among studies based on registry data, and from $4 \%{ }^{14}$ to $6 \%{ }^{25}$ among studies with samples from clinics. The one study that used a population-based asthma sample presented a prevalence ranging between 4 $6 \%{ }^{5}$ Compared to these results, our prevalence of severe asthma at $9.5 \%$ is somewhat higher. However, as we also included those using GINA step 4 treatment, ${ }^{2}$ we expected

A

Severe asthma

B

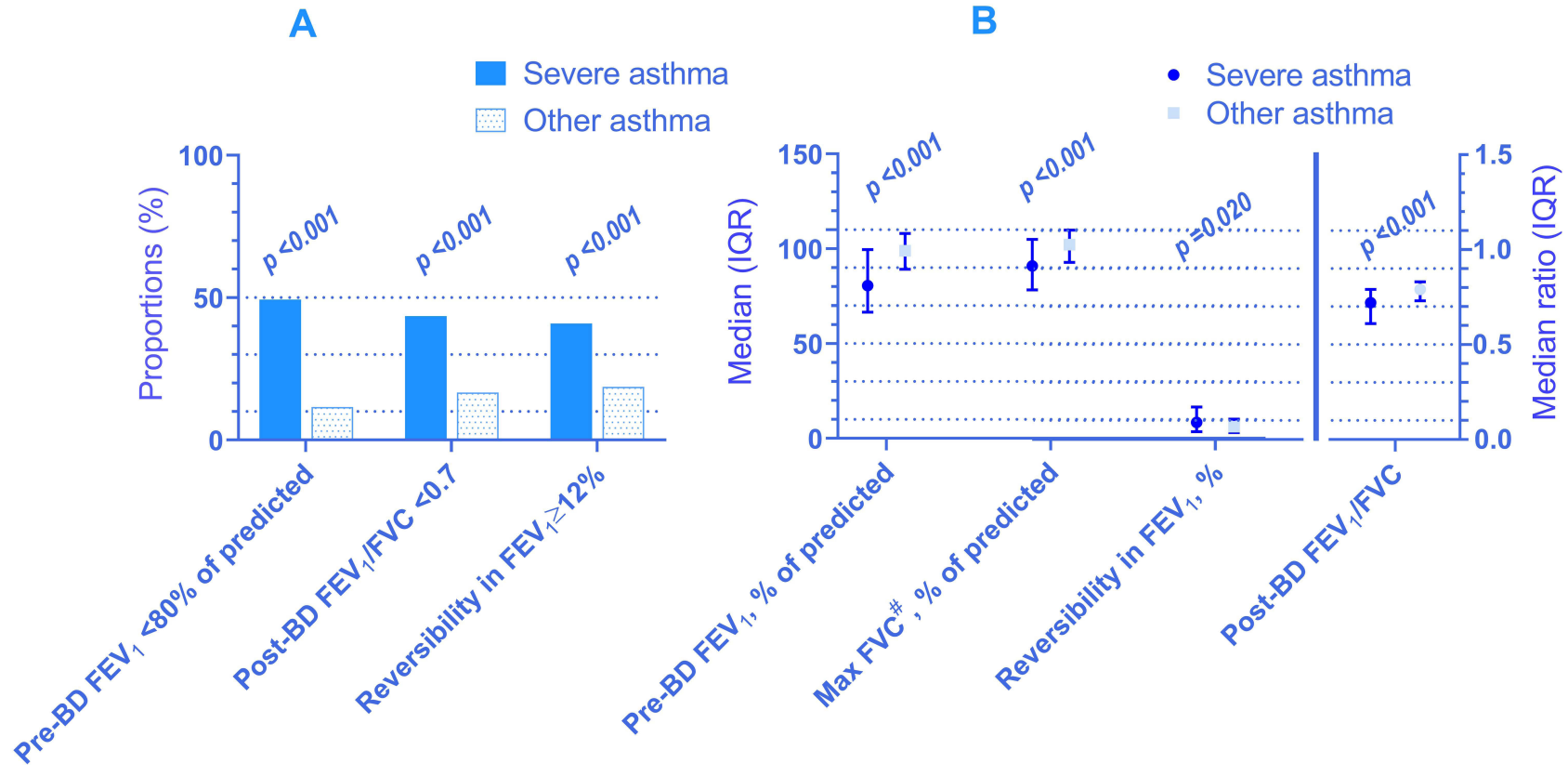

Figure 2 Lung function measurements among individuals with severe vs other asthma in proportions (A) and medians and median ratios (B). Spirometry available in: $\mathrm{n}=735$ (69 severe and 666 other asthma) and 714 for reversibility test ( 66 severe and 648 other asthma). ${ }^{\#} \mathrm{FVC}$ was the only continuous variable that was normally distributed in both of the groups. Mean FVC for severe and other asthma was 91.3 (SD I7.8) and I0I.6 (SD 13.0), respectively. The p-value from the two-tailed independent samples $t$-test however, was not deviating from the Mann-Whitney U-test. For consistency, we present median with IQR and statistical significance from the Mann-Whitney U-test for FVC also.

Abbreviations: $\mathrm{FEV}_{1}$, forced expiratory volume in I s; FVC, forced vital capacity; BD, bronchodilator. 
Table 3 Blood Eosinophil and Neutrophil Levels and Proportion of Patients Showing Blood Eosinophil and Neutrophil Levels Above Different Cut-Point Levels in Severe vs Other Asthma

\begin{tabular}{|c|c|c|c|c|}
\hline Characteristics & & Severe Asthma & Other Asthma & $p$-value \\
\hline Total $n=721$ & & $n=70$ & $n=651$ & \\
\hline Blood eosinophils $\left(\times 10^{9} / \mathrm{L}\right)$ & median (IQR) & $0.2(0.1-0.4)$ & $0.2(0.1-0.3)$ & 0.210 \\
\hline Blood eosinophils $\geq 0.22\left(\times 10^{9} / \mathrm{L}\right)$ & $\%$ & 43.7 & 35.4 & 0.194 \\
\hline Blood eosinophils $\geq 0.3\left(\times 10^{9} / \mathrm{L}\right)$ & $\%$ & 42.3 & 33.3 & 0.148 \\
\hline Blood eosinophils $\geq 0.4\left(\times 10^{9} / \mathrm{L}\right)$ & $\%$ & 23.9 & 17.1 & 0.190 \\
\hline Blood eosinophils $\geq 0.5\left(\times 10^{9} / \mathrm{L}\right)$ & $\%$ & 15.5 & 8.3 & 0.051 \\
\hline Blood eosinophils $\geq 0.6\left(\times 10^{9} / \mathrm{L}\right)$ & $\%$ & 9.9 & 4.2 & a \\
\hline Blood eosinophils $\geq 0.7\left(\times 10^{9} / \mathrm{L}\right)$ & $\%$ & 5.6 & 2.5 & a \\
\hline Blood eosinophils $\geq 0.8\left(\times 10^{9} / \mathrm{L}\right)$ & $\%$ & 2.8 & 1.5 & a \\
\hline Blood neutrophils $\left(\times 10^{9} / \mathrm{L}\right)$ & median (IQR) & $4.4(3.3-5.8)$ & $3.6(2.9-4.6)$ & $<0.001$ \\
\hline Blood neutrophils $\geq 3.0\left(\times 10^{9} / \mathrm{L}\right)$ & $\%$ & 85.9 & 70.3 & 0.005 \\
\hline Blood neutrophils $\geq 4.0\left(\times 10^{9} / \mathrm{L}\right)$ & $\%$ & 62.0 & 38.2 & $<0.001$ \\
\hline Blood neutrophils $\geq 5.0\left(\times 10^{9} / \mathrm{L}\right)$ & $\%$ & 38.0 & 17.4 & $<0.001$ \\
\hline Blood neutrophils $\geq 6.0\left(\times 10^{9} / \mathrm{L}\right)$ & $\%$ & 21.1 & 9.1 & 0.003 \\
\hline Blood neutrophils $\geq 7.0\left(\times 10^{9} / \mathrm{L}\right)$ & $\%$ & 5.6 & 4.3 & a \\
\hline
\end{tabular}

Notes: Data are shown as medians (interquartile range, IQR) and in proportions. Results presented for $n=72 \mathrm{I}$ with data on blood cell counts out of $\mathrm{n}=744$ in the asthma sample. Bold figures indicate statistical significance. ${ }^{a}$ Too few to perform statistical testing.

a higher prevalence due to the expanded criteria. Even higher prevalence of what was referred to as "high-intensity treatment" has been reported. A Dutch study demonstrated a prevalence of difficult-to-control asthma at $17.4 \%$ in their registry-based asthma sample, ${ }^{12}$ and their definition was stricter than what was used in the current study. A British study that also included GINA step 4-users in their sample of potential severe asthma, showed only a marginally smaller prevalence than in our study, at $8 \%$ among asthmatics. ${ }^{16}$

Prevalence of uncontrolled asthma varies among samples categorized as severe asthma, from $30-57 \% 5,6,13-15,27$ up to $90-100 \%,{ }^{7,8,12,25,26}$ and the variation is likely dependent on each study's design and the definition of asthma control. A previous study based on WSAS data, reported prevalence of signs of asthma severity (at 13-36\% among asthmatics), but did not include a specific ICS dose in the definition of severity. ${ }^{28}$ Patients with severe asthma ensure severe limitations in daily life and often normalize their symptoms, ${ }^{29}$ indicating the importance of regular contact with healthcare providers to ensure they receive optimal treatment. Healthcare providers should not only assess asthma control or exacerbation risk, but also investigate possible adherence obstacles, concerns about adverse effects and potential information needs. These strategies are essential for those with severe asthma, as they more often feel alone in their asthma experience compared to those with milder asthma. ${ }^{30}$
GINA guidelines recommend annual follow-ups for patients with any ongoing asthma treatment, ${ }^{2}$ but we found that one-third of those with severe asthma did not have annual contact with a healthcare provider. This finding is supported by another Swedish study of severe asthma, showing even fewer had annual follow-ups. ${ }^{14}$ Among asthmatics in Finland, the follow-ups occurred generally every third year. ${ }^{31}$ However, follow-up visits were recently shown to be more common among those with GINA step 4/5 treatment and those with exacerbations compared to the lower treatment steps and those without exacerbations. ${ }^{32}$ It is vital that all individuals with potentially severe asthma have regular contact with a healthcare provider because asthma symptoms and the risk of exacerbation vary with time. ${ }^{2}$ A previous study using WSAS data showed that annual follow-ups were associated with better adherence and greater belief in the necessity of asthma medication. ${ }^{33}$ Implementation of regular follow-up visits for persons with severe asthma would likely lead to better disease management, ${ }^{2}$ which can lessen impairment in everyday life.

In our sample, those with severe asthma had better lung function compared with existing studies which show a mean $\mathrm{FEV}_{1}$ between $60-72 \%$ of predicted, 3,7,8,16,26,27,34 with some higher reports at $77-81 \%$ of predicted. ${ }^{5,25}$ Most of these studies used clinical samples and generally a higher cut-off for ICS dose, which indicates a higher 


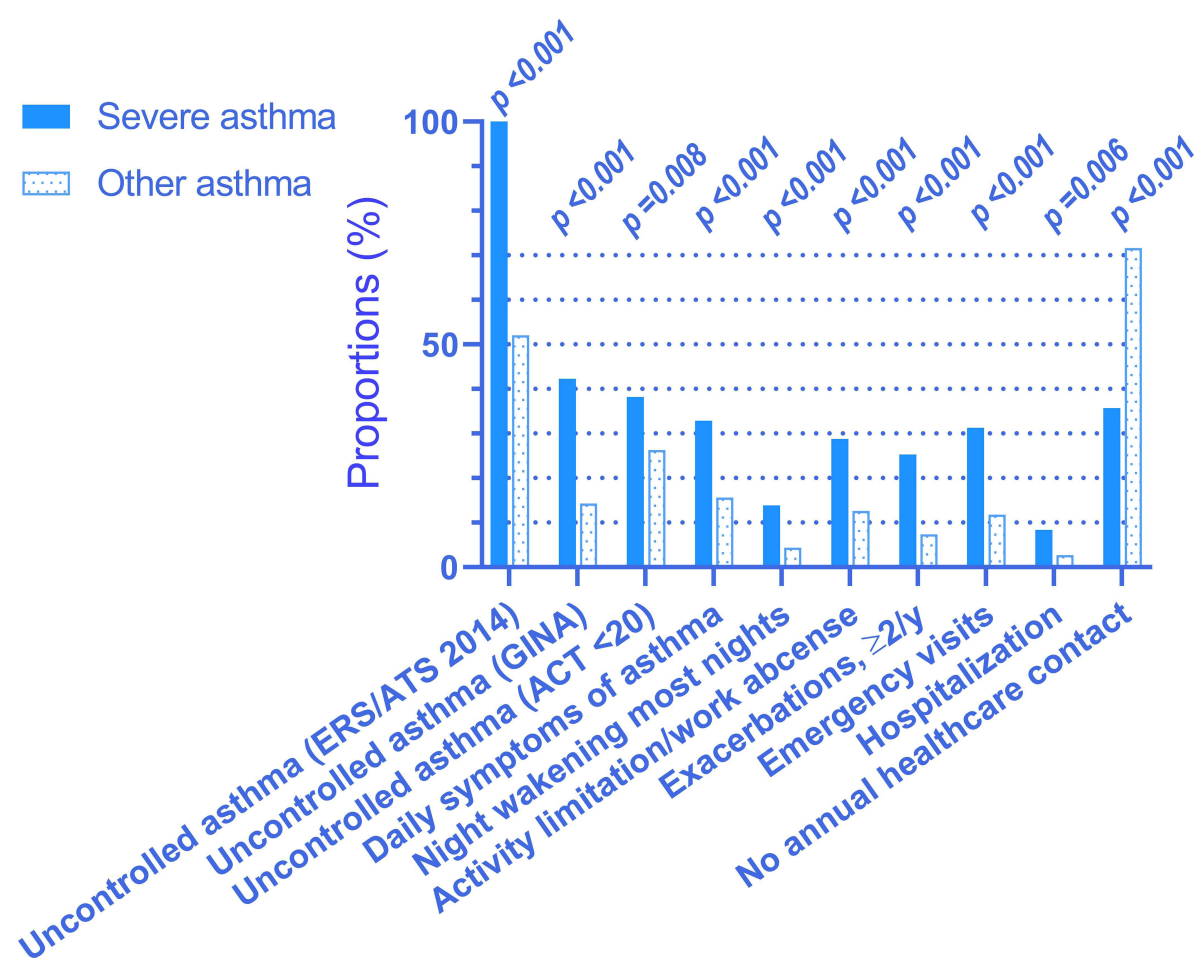

Figure 3 Impact on daily life. Prevalence of symptoms and events within the last 12 months among persons with severe vs other asthma. ACT, Asthma Control Test; only available in $n=432$ (46 severe and 386 other asthma). Responses from remaining variables were available in $84-100 \%$ of asthma sample ( $n=744$ ).

severity of symptoms. Reversibility of $\mathrm{FEV}_{1}$ post-BD was present among over $40 \%$ of individuals with severe asthma in our sample, which suggests that they would probably benefit from additional treatment. However, further increasing ICS dose among those already taking high doses would likely not be beneficial. ${ }^{35}$ Other add-on options or biological drugs would be preferable, none of which were used among our sample at the time of this study.

Previous research has shown higher $^{13}$ or equal levels $3,5,14,25$ of circulating eosinophils in individuals with severe asthma compared to milder asthma, as defined by treatment level. Our results did not show a significant difference in median blood eosinophil count between groups, but those with severe asthma had significantly higher neutrophil levels. This finding is not often reported, but is supported by an increasing number of studies ${ }^{3,5,25}$ and potentially indicates that inflammation in severe asthma is less driven by T-helper type 2 cells (Th2) than in other asthma, or consists of a mixed Th1/Th2 phenotype.

Surprisingly, those with severe asthma reported a much higher prevalence of heart disease compared to those with other asthma; $7.0 \%$ vs $0.4 \%$ respectively reported heart failure. Neither the ERS/ATS ${ }^{1}$ nor GINA ${ }^{2}$ reports cardiovascular-related comorbidities within this group. However, similar results have been shown in other recent studies, ${ }^{14,16}$ but not with such a large difference compared to mild/ moderate asthma. We suggest this cannot be explained merely by higher age among those with severe asthma or by the small difference in smoking history between the groups. An association between cardiovascular diseases and adult-onset asthma has been described, especially among women, and also with high use of corticosteroids and beta $_{2}$-agonists. ${ }^{36}$ The latter is by definition more common among persons with severe asthma. ${ }^{1,2}$ However, as the proportion of late-onset asthma was larger among those with severe compared to other asthma, we believe further research is needed to explore this association.

\section{Strengths and Limitations of the Study}

The foremost strengths of our study are the populationbased design with a large randomly selected sample and the large age range of participants. Another strength is the combination of structured interviews and self-administered questionnaires with clinical examinations, which enabled a comprehensive investigation. Additionally, skilled nurses 
and biomedical scientists performed all procedures, minimizing interviewer bias. To further limit possible bias, all spirometry results were verified by one medical doctor.

One limitation of this study is that adherence to prescribed medication was assessed by self-report. However, all participants were encouraged to answer honestly, not based on what they should be doing based on recommendations by healthcare providers. As a result, 35 individuals were excluded from the severe asthma group because they responded "occasionally" instead of "most days" to the frequency of medication use.

Another potential weakness is that some participants might have coexisting Chronic Obstructive Pulmonary Disease (COPD). If those with a smoking history of $>10$ pack-years and an obstructive spirometry $(n=20)$ were excluded, $72 \%$ would remain in the severe asthma group. Since some individuals with asthma are known to have a persistent airflow limitation, ${ }^{1,37}$ we did not exclude these individuals, as they also had current asthma.

One limitation may be that the data was collected between 2009-2012, ie before the era of current biologic treatments of severe asthma and that evaluation of severe asthma has developed since that time. However, the definition of severe asthma used in the current study is up to date and we had relevant data in use to evaluate severe asthma. Patients currently eligible for biologic asthma therapy would have been included in the group of severe asthma. Furthermore, the results are of value as population-based studies on severe asthma are rare and most other studies are derived from highly selected severe asthma cohorts.

Additionally, since the cross-sectional study design does not provide the opportunity to treat and evaluate the participants as preferred, ${ }^{1,2,17}$ there is a risk of overestimating the prevalence of severe asthma. However, this risk may always exist, as studies have shown that it can be challenging to differentiate between difficult-to-treat and severe asthma even when attempted. ${ }^{12,38}$

\section{Conclusion}

The $9.5 \%$ prevalence of severe asthma among asthmatics was higher than in previous studies, which were often based on registries and clinics with a higher cut-off for ICS dose. Our prevalence of $1.1 \%$ in the random sample would correspond to approximately 83,000 individuals in the Swedish population. Considering that many suffer from a large burden of impairments and symptoms and that they also generate high costs for society, we suggest that healthcare providers implement strategies to ensure annual follow-ups, which were lacking according to the current study. To manage this deficiency we suggest future studies of its causes among patients as well as from the society's point of view. Furthermore, since asthma intensity varies over time, a more preventive approach would benefit both patients and society.

\section{Acknowledgments}

We would like to acknowledge The Herman Krefting Foundation for Allergy and Asthma Research, the Swedish Heart-Lung Foundation and the ALF-agreement between the Swedish government and the Västra Götaland Regional Council for unrestricted funding of the study. We also thank the study staff and participants for their involvement in the study.

\section{Disclosure}

Ms Lina Rönnebjerg reports personal fees from AstraZeneca, outside the submitted work. Professor Hannu Kankaanranta reports personal fees, non-financial support from AstraZeneca, Orion Pharma; personal fees from BoehringerIngelheim, Chiesi Pharma, GlaxoSmithKline, MSD, Mundipharma, Novartis, and Sanofi, outside the submitted work. Dr Helena Backman reports personal fees from Astra Zeneca and Boehringer Ingelheim, outside the submitted work. Professor Bo Lundbäck reports grants from ThermoFisher, personal fees from Sanofi, outside the submitted work. The authors report no other conflicts of interest in this work.

\section{References}

1. Chung KF, Wenzel SE, Brozek JL, et al. International ERS/ATS guidelines on definition, evaluation and treatment of severe asthma. Eur Respir J. 2014;43(2):343-373. doi:10.1183/09031936.00202013

2. Global Initiative for Asthma. Global strategy for asthma management and prevention. Available from: www.ginasthma.org/gina-reports/. Accessed October 132020

3. Shaw DE, Sousa AR, Fowler SJ, et al. Clinical and inflammatory characteristics of the European U-BIOPRED adult severe asthma cohort. Eur Respir J. 2015;46(5):1308-1321. doi:10.1183/ 13993003.00779-2015

4. Aubier M, Thabut G, Fabry-Vendrand C. Characteristics of patients with severe, uncontrolled, eosinophilic asthma enrolled in a French cohort. $J$ Asthma Allergy. 2018;11:217-224. doi:10.2147/JAA. S170866

5. Backman H, Jansson SA, Stridsman C, et al. Severe asthma-A population study perspective. Clin Exp Allergy. 2019;49 (6):819-828. doi:10.1111/cea.13378

6. von Bulow A, Kriegbaum M, Backer V, Porsbjerg C. The prevalence of severe asthma and low asthma control among Danish adults. J Allergy Clin Immunol Pract. 2014;2(6):759-767. doi:10.1016/j. jaip.2014.05.005 
7. Schleich F, Brusselle G, Louis R, et al. Heterogeneity of phenotypes in severe asthmatics. The Belgian Severe Asthma Registry (BSAR). Respir Med. 2014;108(12):1723-1732. doi:10.1016/j.rmed.2014.10.007

8. Mullerova H, Cockle SM, Gunsoy NB, Nelsen LM, Albers FC. Clinical characteristics and burden of illness among adolescent and adult patients with severe asthma by asthma control: the IDEAL study. $J$ Asthma. 2021;58(4):459-470. doi:10.1080/02770903.2019.1708095

9. Antonicelli L, Bucca C, Neri M, et al. Asthma severity and medical resource utilisation. Eur Respir J. 2004;23(5):723-729. doi:10.1183/ 09031936.04.00004904

10. Zeiger RS, Schatz M, Dalal AA, et al. Utilization and Costs of Severe Uncontrolled Asthma in a Managed-Care Setting. J Allergy Clin Immunol Pract. 2016;4(1):120-9 e3. doi:10.1016/j.jaip.2015.08.003

11. Sadatsafavi M, Lynd L, Marra C, et al. Direct health care costs associated with asthma in British Columbia. Can Respir J. 2010;17 (2):74-80. doi:10.1155/2010/361071

12. Hekking PW, Wener RR, Amelink M, Zwinderman AH, Bouvy ML, Bel EH. The prevalence of severe refractory asthma. J Allergy Clin Immunol. 2015;135(4):896-902. doi:10.1016/j.jaci.2014.08.042

13. Varsano S, Segev D, Shitrit D. Severe and non-severe asthma in the community: a large electronic database analysis. Respir Med. 2017;123:131-139. doi:10.1016/j.rmed.2016.12.017

14. Larsson K, Stallberg B, Lisspers K, et al. Prevalence and management of severe asthma in primary care: an observational cohort study in Sweden (PACEHR). Respir Res. 2018;19(1):12. doi:10.1186/ s12931-018-0719-x

15. Nagase H, Adachi M, Matsunaga K, et al. Prevalence, disease burden, and treatment reality of patients with severe, uncontrolled asthma in Japan. Allergol Int. 2020;69(1):53-60. doi:10.1016/j. alit.2019.06.003

16. Ryan D, Heatley H, Heaney LG, et al. Potential Severe Asthma Hidden in UK Primary Care. J Allergy Clin Immunol Pract. 2021;9 (4):1612-1623 e9. doi:10.1016/j.jaip.2020.11.053

17. Porsbjerg C, Ulrik C, Skjold T, et al. Nordic consensus statement on the systematic assessment and management of possible severe asthma in adults. Eur Clin Respir J. 2018;5(1):1440868. doi:10.1080/ 20018525.2018.1440868

18. Ekerljung L, Bossios A, Lotvall J, et al. Multi-symptom asthma as an indication of disease severity in epidemiology. Eur Respir J. 2011;38 (4):825-832. doi:10.1183/09031936.00143710

19. Lotvall J, Ekerljung L, Ronmark EP, et al. West Sweden Asthma Study: prevalence trends over the last 18 years argues no recent increase in asthma. Respir Res. 2009;10:94. doi:10.1186/1465-9921-10-94

20. Nwaru BI, Ekerljung L, Radinger M, et al. Cohort profile: the West Sweden Asthma Study (WSAS): a multidisciplinary population-based longitudinal study of asthma, allergy and respiratory conditions in adults. BMJ Open. 2019;9(6):e027808. doi:10.1136/bmjopen-2018027808

21. Rönmark EP, Ekerljung L, Lötvall J, Torén K, Rönmark E, Lundbäck B. Large scale questionnaire survey on respiratory health in Sweden: effects of late- and non-response. Respir Med. 2009;103 (12):1807-1815. doi:10.1016/j.rmed.2009.07.014

22. World Medical Association Declaration of Helsinki. Ethical principles for medical research involving human subjects. Available from: https://www.wma.net/what-we-do/medical-ethics/declaration-ofhelsinki/. Accessed August 182021.

23. Global Initiative for Asthma. Global strategy for asthma management and prevention. Available from: www.ginasthma.org/archived-reports /. Accessed October 132020.
24. Kosinski MBM, Turner-Bowker DM, Fortin EW. Asthma Control Test ${ }^{\mathrm{TM}}$ : A User's Guide. QualityMetric Incorporated; 2004.

25. Ilmarinen P, Tuomisto LE, Niemela O, Kankaanranta H. Prevalence of Patients Eligible for Anti-IL-5 Treatment in a Cohort of Adult-Onset Asthma. J Allergy Clin Immunol Pract. 2019;7(1):165174 e4. doi:10.1016/j.jaip.2018.05.032

26. Csoma Z, Gal Z, Gezsi A, Herjavecz I, Szalai C. Prevalence and characterization of severe asthma in Hungary. Sci Rep. 2020;10 (1):9274. doi:10.1038/s41598-020-66445-4

27. Wang E, Wechsler ME, Tran TN, et al. Characterization of Severe Asthma Worldwide: data From the International Severe Asthma Registry. Chest. 2020;157(4):790-804. doi:10.1016/j.chest.20 19.10.053

28. Mincheva R, Ekerljung L, Bossios A, Lundback B, Lotvall J. High prevalence of severe asthma in a large random population study. J Allergy Clin Immunol. 2018;141(6):2256-2264 e2. doi:10.1016/j. jaci.2017.07.047

29. Bidad N, Barnes N, Griffiths C, Horne R. Understanding patients' perceptions of asthma control: a qualitative study. Eur Respir J. 2018;51(6):1701346. doi:10.1183/13993003.01346-2017

30. Foster JM, McDonald VM, Guo M, Reddel HK. "I have lost in every facet of my life": the hidden burden of severe asthma. Eur Respir J. 2017;50(3):1700765. doi:10.1183/13993003.00765-2017

31. Takala J, Ilmarinen P, Tuomisto LE, Vahatalo I, Niemela O, Kankaanranta H. Planned primary health care asthma contacts during 12-year follow-up after Finnish National Asthma Programme: focus on spirometry. NPJ Prim Care Respir Med. 2020;30(1):8. doi:10.1038/s41533-020-0166-2

32. Stridsman C, Axelsson M, Warm K, Backman H. Uncontrolled asthma occurs in all GINA treatment steps and is associated with worse physical health - A report from the OLIN adult asthma cohort. J Asthma. 2021;58(5):586-595. doi:10.1080/02770903.2020.1713150

33. Axelsson M, Ekerljung L, Lundback B. The Significance of Asthma Follow-Up Consultations for Adherence to Asthma Medication, Asthma Medication Beliefs, and Asthma Control. Nurs Res Pract. 2015;2015:139070.

34. van Bragt J, Adcock IM, Bel EHD, et al. Characteristics and treatment regimens across ERS SHARP severe asthma registries. Eur Respir J. 2020;55(1):urn:issn:0903-1936. doi:10.1183/13993003.01 163-2019

35. Kankaanranta H, Lahdensuo A, Moilanen E, Barnes PJ. Add-on therapy options in asthma not adequately controlled by inhaled corticosteroids: a comprehensive review. Respir Res. 2004;5(1):17. doi:10.1186/1465-9921-5-17

36. Kankaanranta H, Kauppi P, Tuomisto LE, Ilmarinen P. Emerging Comorbidities in Adult Asthma: risks, Clinical Associations, and Mechanisms. Mediators Inflamm. 2016;2016:3690628. doi:10.1155/ 2016/3690628

37. Ekerljung L, Mincheva R, Hagstad S, et al. Prevalence, clinical characteristics and morbidity of the Asthma-COPD overlap in a general population sample. $J$ Asthma. 2018;55(5):461-469. doi:10.1080/02770903.2017.1339799

38. von Bulow A, Backer V, Bodtger U, et al. Differentiation of adult severe asthma from difficult-to-treat asthma - Outcomes of a systematic assessment protocol. Respir Med. 2018;145:41-47. doi:10.1016/j.rmed.2018.10.020 


\section{Publish your work in this journal}

The Journal of Asthma and Allergy is an international, peer-reviewed open-access journal publishing original research, reports, editorials and commentaries on the following topics: Asthma; Pulmonary physiology; Asthma related clinical health; Clinical immunology and the immunological basis of disease; Pharmacological interventions and new therapies. The manuscript management system is completely online and includes a very quick and fair peer-review system, which is all easy to use. Visit http://www.dovepress.com/testimonials.php to read real quotes from published authors.

Submit your manuscript here: https://www.dovepress.com/journal-of-asthma-and-allergy-journal 